\title{
Análisis de las Proyecciones de Crecimiento Económico del Banco Central de Chile: 1991-2017
}

\author{
Sergio Zuniga-Jara ${ }^{1}$, Oscar Sjoberg-Tapia ${ }^{2}$ y Diego Opazo-Gallardo ${ }^{1}$ \\ (1) Escuela de Cs. Empresariales, Universidad Católica del Norte, Larrondo 1281, Coquimbo \\ (2) Facultad de Cs. Empresariales, Universidad Arturo Prat, Av. Arturo Prat 2120, Iquique \\ E-mail: sz@ucn.cl; osjoberg@unap.cl; diego.opazogallardo@gmail.com
}

Recibido May. 18, 2018; Aceptado Jul. 27, 2018; Versión final Ago. 30, 2018, Publicado Feb. 2019

\begin{abstract}
Resumen
En este estudio se comparó las predicciones de crecimiento económico para el periodo 1991-2016, con el crecimiento real ex-post del PIB chileno, basado en la información entregada por el Banco Central de Chile. Esta institución publica regularmente predicciones de crecimiento del PIB de Chile. Anteriormente lo hizo en los Informes de Inflación al Senado, y actualmente lo hace en los Informe de Política Monetaria. Los resultados de las medidas de precisión indican que los errores medios incurridos por el Banco Central fueron mayores en las predicciones de más largo plazo, tal como se esperaba. Además, se encontró que en las predicciones de corto plazo existió sesgos promedio pesimistas, mientras que en las de largo plazo, el sesgo fue optimista. Finalmente, se encuentra que si bien las predicciones realizadas por el Banco Central en el periodo 1991-2008 fueron menos precisas, en promedio fueron insesgadas y eficientes, a diferencia de las predicciones realizadas en el periodo 2009-2016, las que mostraron poseer un sesgo optimista estadísticamente significativo.
\end{abstract}

Palabras claves: crecimiento del PIB; pronósticos; Banco Central de Chile; insesgamiento; eficiencia; precisión

\section{Analysis of the Economic Growth Forecast from the Central Bank of Chile: 1991-2017}

\begin{abstract}
In this study, the predictions of the economic growth (1991-2016) with the real ex-post growth of the Chilean GDP were analyzed, based on the information of the Central Bank of Chile. This institution regularly publishes forecasts of Chile's GDP growth. Previously they did it in the Inflation Reports to the Senate, and currently in the Monetary Policy Report. The results of the precision measurements indicate that the mean errors were higher in the long-term predictions, as expected. In addition, in the short-term predictions a pessimistic average bias was observed, while in the long-term the bias was optimistic. Finally, although the predictions made in 1991-2008 were less precise, on the average, they were unbiased and efficient, unlike the predictions made in 2009-2016, which showed to have a statistically significant optimistic bias.
\end{abstract}

Keywords: forecast; GDP growth; forecasts; Central Bank of Chile; unbiasedness; efficiency; accuracy 


\section{INTRODUCCIÓN}

Para planificar sus decisiones, los agentes económicos (empresas, familias y gobiernos) requieren de proyecciones del crecimiento económico nacional. Los bancos centrales habitualmente generan este tipo de proyecciones. En el caso de Chile, el Banco Central de Chile (BCCh), a través del Informe de Política Monetaria (IPoM) informa al Senado, al Gobierno y al público general sobre la evolución de la inflación, las consecuencias de las políticas monetarias y el comportamiento del Producto Interno Bruto (PIB). Esta variable es utilizada por el Gobierno para la planificación de políticas fiscales, y por el propio BCCh para diseñar políticas monetarias. Sin embargo los pronósticos del PIB pueden enfrentar problemas de baja precisión, sesgos e ineficiencia, lo que puede inducir a tomar decisiones basadas en información errónea.

El análisis de la calidad de las predicciones económicas es un campo en el cual existe bastante investigación. En particular, a nivel mundial se ha analizado no solo las proyecciones de crecimiento económico, sino que también de inflación, tipo de cambio y desempleo, entre otras. La mayoría de los estudios están orientados en determinar la existencia de sesgo y/o eficiencia en los pronósticos, y en general se concluye que en el corto plazo las predicciones son más precisas e insesgadas. Lahiri (2010) evaluó los pronósticos del PIB para 18 países pertenecientes a la Organización para la Cooperación y el Desarrollo Económico (OCDE). Los resultados mostraron que los pronósticos con un horizonte de tiempo menor a un año y medio eran de calidad, mientras que en caso contrario, los pronósticos superiores a dos años no son fiables. The Bank of England (2015) evaluó sus propias predicciones de crecimiento económico, no encontrando evidencia significativa de sesgo e ineficiencia, y comparándose favorablemente respecto a las predicciones de otros bancos centrales. Para los pronósticos de la Fed en los EEUU, Sinclair, Stekler y Joutz (2008) encontraron que los pronósticos a un trimestre no demostraron evidencia de sesgo e ineficiencia, sin embargo a plazos mayores encontraron una predicción sesgada del PIB, siendo subvalorado en expansiones y sobrevalorado en recesiones.

En Chile, se dispone sólo de dos artículos que han analizado la calidad de los pronósticos del BCCh del PIB:

1.- Albagli et al. (2003), quienes evalúan la precisión de las proyecciones de actividad económica e inflación provenientes del informe al Senado (desde 1990) y del IPoM (desde el año 2000). A través del error cuadrático medio (ECM) y la raíz del error cuadrático medio (RECM) concluyen que las predicciones elaboradas después del año 2000 fueron más precisas. También realizaron un benchmarking comparativo de los pronósticos del BCCh, Consensus Forecasts y de los Bancos Centrales de Inglaterra, Suecia, Nueva Zelandia, Brasil y Canadá. Concluyeron que el desempeño de las predicciones del BCCh es similar al de los otros Bancos. No obstante, los pronósticos privados mostraron ser marginalmente superiores en comparación al BCCh. 2.- Pincheira (2010) consideró dos horizontes de pronósticos; el primero calculado en septiembre para el final del mismo año, y el segundo calculado en el mismo periodo para el final de año subsiguiente. Además, integró un benchmarking con predicciones de diferentes modelos auto-regresivos y de pronósticos de instituciones privadas, utilizando como criterio el mean absolute prediction error (MAPE). Sus resultados fueron mixtos, a favor y en contra de los pronósticos del BCCh, sugiriendo sin embargo que existe espacio para que los mismos sean mejorados.

Con el objetivo de ampliar y actualizar tales resultados, en el presente estudio analizamos la calidad de las proyecciones de crecimiento del PIB realizadas por el BCCh, pero usando ahora el total de datos disponibles hasta este momento, y siguiendo un enfoque más clásico. Es decir, estimando directamente la precisión (Accuracy), insesgamiento (Unbiasedness) y eficiencia (Efficiency) de tales proyecciones de crecimiento. Para ello, a continuación se discute la metodología a utilizar para responder la interrogante central. Luego se presentan los resultados, para finalmente discutir y concluir sobre los mismos y sus implicancias.

\section{METODOLOGÍA}

Existen varios enfoques para evaluar la calidad de los pronósticos económicos (Holden et al., 1990 y Higgins et al., 2011, Bank of England, 2015). Aquí seguimos los enfoques clásicos, los que se centran en medir precisión, insesgamiento y eficiencia como propiedades deseables en las estimaciones. El insesgamiento se refiere a si los pronósticos han sido persistentemente demasiado optimistas o pesimistas. Por otro lado, la eficiencia mide si los pronósticos han reflejado toda la información disponible relevante en el momento en que se hicieron. Entonces, una predicción es eficiente si, por ejemplo, el uso del historial de errores de predicción (el récord histórico de errores), no permite mejorar las predicciones de los periodos siguientes. 
La base de datos contiene los pares de datos de predicciones de crecimiento del PIB y valor efectivo expost del mismo, para cada uno de los diferentes horizontes en los que el BCCh hizo una predicción. 1) Los pronósticos del BCCh se obtuvieron de dos fuentes (Tabla 1): Las proyecciones del Informe de Inflación al Senado, entre los años 1991-2000 (Pincheira, 2010). Este informe se publicó en septiembre de cada año, con las proyecciones del PIB para el final del año en curso y el final del año siguiente. También de las proyecciones publicadas en IPoM a partir del año 2000. El IPoM del año 2000 se publicó en mayo y septiembre; en los años 2001-2008 fue publicado cuatrimestralmente (enero, mayo y septiembre de cada año), y a partir del año 2009 comenzó a publicarse trimestralmente (marzo, junio, septiembre y diciembre). 2) Las proyecciones del PIB observado o realizado. Se obtuvieron del BCCh, como la variación anual de su gasto, a precios del año anterior encadenado, utilizando de referencia el año 2013.

Tabla 1: Características de la base de datos de predicciones. ( Indica estimaciones realizadas esporádicamente)

\begin{tabular}{|c|c|c|c|}
\hline & Periodicidad & $\begin{array}{c}\text { Horizonte } \\
\text { (pasos hacia adelante) }\end{array}$ & $\begin{array}{c}\text { Numero de } \\
\text { predicciones disponibles }\end{array}$ \\
\hline $1991-1999$ & Anual & 3 y 15 meses & 18 \\
\hline $2000-2008$ & Cuatrimestral & $3-7-11-15-19^{*}-23^{*}-27^{*}$ meses & 43 \\
\hline $2009-2016$ & Trimestral & $0-3-6-9-12-15-18^{*}$ meses & 50 \\
\hline Totales & & & 111 \\
\hline
\end{tabular}

\section{Precisión de las Predicciones}

Sea $A_{p}$ el valor realizado (observado, post-evento) de crecimiento del PIB, el cual se desea predecir. Ap es siempre anual, y finaliza el último día de cada año, es decir se refiere a un año corriente. Sea $h$ el número de meses previos al término del periodo anual que se desea predecir, es decir $h$ es el horizonte de predicción, en número de meses. Sea $F_{t+h, p}$ la predicción realizada el año t, con $\mathrm{h}$ meses de anticipación antes de que $A_{p}$ sea realizado. $p$ indica el año cuyo crecimiento del PIB se quiere predecir. $A$ modo de ilustración, $F_{2016+21,2017}$, indica un pronóstico realizado $\mathrm{h}=21$ meses antes de que termine el año $\mathrm{p}=2017$, es decir este pronóstico se realizó en marzo de $\mathrm{t}=2016$, debido a que ahí faltan $\mathrm{h}=21$ meses para que finalice el año $\mathrm{p}=2017$. En general, el proceso de evaluación de la precisión, insesgamiento y eficiencia consiste en comparar los valores de $A_{p}$ (el PIB observado) con los de $F_{t+h, p}$ (el PIB pronosticado). Los errores de predicción vienen dados por la diferencia entre ambos valores: $e_{t+h, p}=A_{p}-F_{t+h, p}$.

Existen varios indicadores de precisión de las predicciones, tal que un valor cercano a 0 implica un pronóstico preciso. De ellos se han seleccionado los siguientes (Villada, Muñoz y García-Quintero, 2016), los que se estimaron en distintos periodos, sub-periodos y horizontes de predicción:

Error Medio: $M E=\frac{1}{T} \sum_{t=1}^{T} e_{t+h, t}$;

Error Medio Absoluto: $\boldsymbol{M A E}=\frac{1}{T} \sum_{t=1}^{T}\left|e_{t+h, t}\right|$

Raíz del Error Cuadrático Medio: $R M S E=\sqrt{\frac{1}{T} \sum_{t=1}^{T}\left(e_{t+h, t}\right)^{2}}$

Tanto MAE como RMSE expresan el error de predicción del modelo promedio en unidades de la variable de interés. Ambas métricas pueden variar de 0 a $\infty$ y son indiferentes del signo de los errores. Son puntuaciones negativamente orientadas, lo que significa que los valores más bajos son mejores. RMSE da un peso relativamente alto a grandes errores, y puede ser más útil cuando los grandes errores son particularmente indeseables.

\section{Insesgamiento y Eficiencia de las Predicciones}

Theil (1966) y Mincer y Zarnowitz (1969) plantearon una regresión donde el valor realizado expost $\left(A_{t}\right)$, es explicado por su predicción exante $\left(F_{t}\right)$. La regresión con horizontes de predicción es:

$A_{p}=\beta_{0}+\beta_{1} F_{t+h, p}+e_{t+h, p}$

El rechazo de la siguiente hipótesis nula conjunta: $H_{0}: \beta_{0}=0$ y $H_{0}: \beta_{1}=1$ indica la presencia de sesgo débil e ineficiencia débil en los pronósticos. El P-value del test conjunto es informado en el estadístico $\mathrm{F}$ del test Wald para las restricciones de los coeficientes, y el procedimiento de Newey y West (1987) es utilizado para 
estimar los errores estándar consistentes corregidos por heterocedasticidad y auto-correlación (HAC). En caso de no rechazo de la hipótesis conjunta, se realiza un test de sesgo débil y eficiencia débil de forma separada, de modo que el rechazo individual de $H_{0}: \beta_{0}=0$ determina sesgo en los pronósticos, y el rechazo individual de $H_{0}: \beta_{1}=1$ indica ineficiencia de los pronósticos. Esto, debido a que cuanto mayor es la desviación de la pendiente de la regresión de la unidad, menos eficiente es el pronóstico. La Figura 1 ilustra lo anterior, mostrando $F_{t+h, p}$ en el eje horizontal, versus $A_{p}$ en el eje vertical. Si el BCCh acierta en cada una de sus predicciones, entonces se tendría una línea de $45^{\circ}$ desde el origen (la línea de predicción perfecta). Puede notarse que La varianza alrededor de LPF (Línea de predicciones perfectas) es el error cuadrático medio del pronóstico.

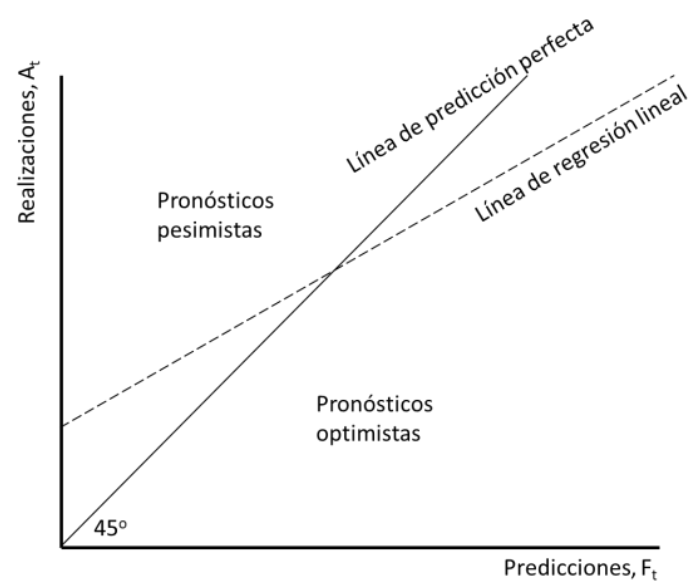

Fig.1: Método clásico de estimación de insesgamiento y eficiencia de las predicciones.

Fuente: Adaptado de Mincer \& Zarnowitz (1969).

\section{Insegamiento y Eficiencia Fuerte de los Pronósticos}

Una versión corregida para sesgo (insesgamiento fuerte) surge dadas las críticas de Webb (1987) y Mankiw and Shapiro (1986) al enfoque anteriormente expuesto. Para resolver esto, Croushore (2010) plantea un test de media cero de los errores, vía una regresión donde los errores de predicción son explicados por el intercepto de la Ec. (1), es decir $e_{t+h, p}=\beta_{0}+u_{t+h, p}$, donde $e_{t+h, p}$ representa el error de proyección h pasos hacia adelante, $\beta_{0}$ es la constante a estimar y $u_{t+h, p}$ es un término de error medio cero, bien comportado. La hipótesis nula de insesgamiento (fuerte) es $H_{0}: \beta_{0}=0$. La regresión se estima por MCO y con errores consistentes por Newey y West (1987).

Una prueba de eficiencia fuerte es proporcionada por el Bank of England (2015), la que sugiere regresionar los errores de pronóstico en la información que se conocía cuando se hicieron los pronósticos. Idealmente, los errores de pronóstico no estarán correlacionados con dicha información, o los errores podrían haberse reducido al incorporar esa información cuando se hicieron los pronósticos. Para realizar pruebas de eficiencia fuerte, estimamos la siguiente ecuación usando MCO con errores estándar HAC:

$$
e_{t+h, p}=\gamma_{0}+\gamma_{1} z_{t+h, p}+u_{t+h, p}
$$

donde e es el error de pronóstico como se definió anteriormente, $\mathbf{z}$ es una variable conocida por el pronosticador en el momento de hacer la predicción, y u es un término de error bien comportado. Bajo la hipótesis nula de la eficiencia fuerte, probamos si $\gamma_{1}=0$. En nuestro caso, $\mathbf{z}$ fue definido como el error de pronóstico previo, conocida por los pronosticadores.

\section{RESULTADOS}

En base a lo anterior se construyó la base de datos. Cuando el BCCh publicó pronósticos con rangos, se utilizó el promedio como pronóstico. La primera columna contiene las fechas de publicación de los Informes de Inflación al Senado (antes del año 2000) y los del Informe de Política Monetaria (después del año 2000).

\section{Errores de Predicción: Visión gráfica}

La Fig. 2 compara la línea de predicción perfecta $\left(45^{\circ}\right)$ con la línea de predicción real para el periodo completo, en todos los horizontes de predicción. Globalmente se puede apreciar que la línea de $45^{\circ}$ se encuentra casi siempre sobre la línea de regresión, lo cual sugiere la predominancia de estimaciones 
optimistas. Además, se aprecia globalmente una diferencia en ambos interceptos, lo cual puede indicar la existencia de sesgo. Esto se analizará estadísticamente más adelante.

La Fig. 3a y 3b muestra la nube de dispersión de los pronósticos y sus realizaciones para el corto plazo y largo plazo respectivamente, independiente de la fecha en que se realizaron tales pronósticos. Se consideró corto plazo a los pronósticos realizados con horizontes de 3, 6 y 7 meses, y como horizonte de largo plazo los de 11, 12 y 15 meses. Los puntos en la Fig. 3b (largo plazo) presentan mayor dispersión que los de la Fig. 3a, respecto a la línea central, lo que sugiere que los errores de predicción aumentan al aumentar el horizonte de predicción, algo que debería ser esperado a priori. Además, en la Fig. 3a puede observarse una forma de convergencia a la media en las predicciones, en el sentido de que cuando el crecimiento es relativamente alto, las predicciones tienden a ser pesimistas (la línea de regresión se encuentra arriba de $45^{\circ}$ ). A la inversa, cuando el crecimiento es bajo, las predicciones fueron optimistas. Finalmente, las Figuras sugieren que las predicciones de largo plazo, si bien presentan mayor dispersión, parecen ajustarse mejor en promedio que las predicciones de corto plazo, debido a que la pendiente de la línea de predicción parece acercarse más a la de predicción perfecta que en el caso de largo plazo.

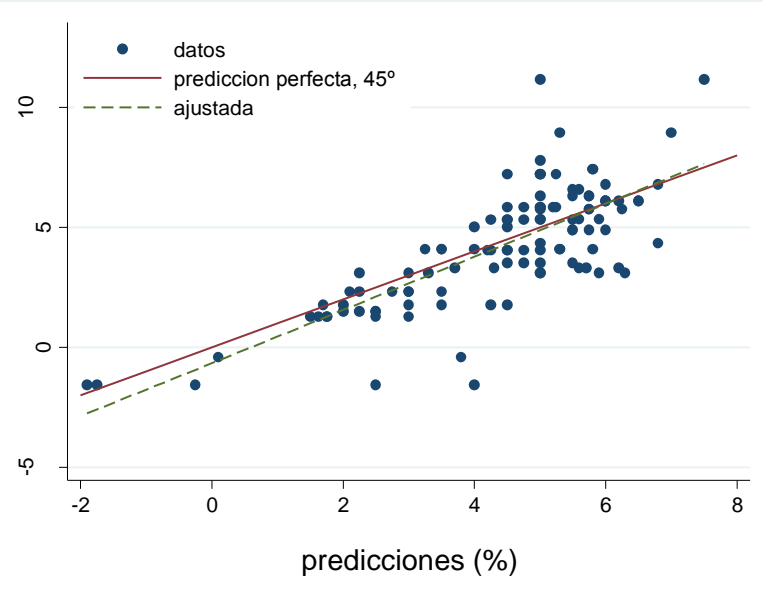

Fig. 2: Predicciones versus realizaciones, todos los horizontes, periodo completo, 1991-2016 (111 observaciones).

Fig. 3a. Corto plazo 03_06_07 meses

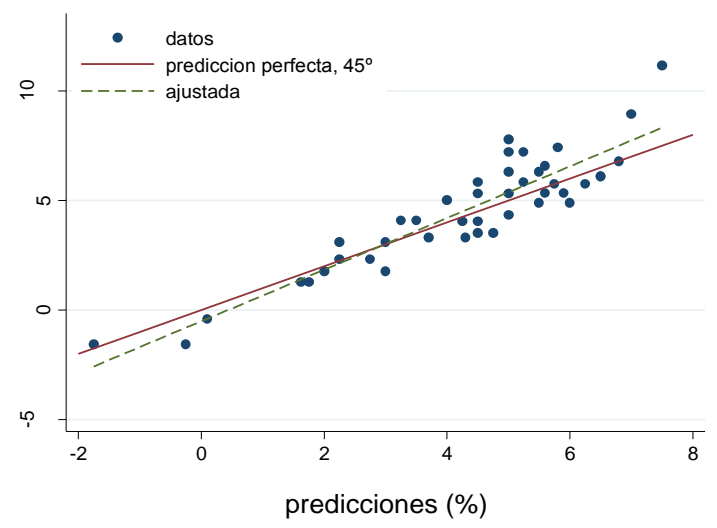

Fig. 3b Largo plazo. 11_12_15 meses

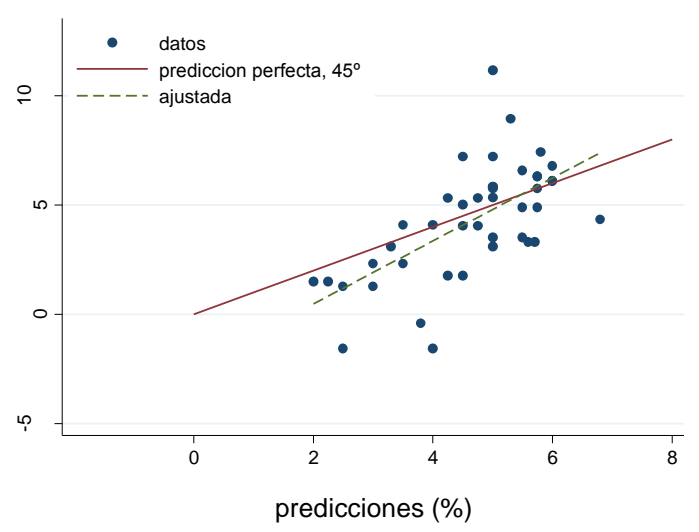

Fig. 3: Predicciones versus realizaciones, por horizonte de predicción, periodo completo, 1991-2016.

Las Fig. 4a y 4b muestran las predicciones realizadas en el periodo 1991-2008 (cuando se publicaban predicciones anualmente y cuatrimestralmente), en comparación con las realizadas más recientemente en el periodo 2009-2016 (cuando comenzaron a publicarse predicciones trimestrales). La Fig. 4a muestra un número de puntos alineados verticalmente en una predicción de $5 \%$, indicando que este nivel de crecimiento económico predominó en las predicciones de ese periodo. Por el contrario, en la Fig. 4b se aprecian varios puntos alineados horizontalmente, indicando que para un mismo año existieron varias predicciones, lo que se explica en el hecho de que en ese periodo se publicó 4 predicciones por año (hubo varias predicciones para un mismo periodo, cuyo valor realizado de crecimiento ex post fue el mismo).

La Fig. 4a sugiere que las predicciones realizadas en 1991-2008 presentaron mayores errores en magnitud (mayor dispersión de puntos alrededor de la línea de predicción), en comparación con las predicciones realizadas en el periodo 2009-20016. Adicionalmente, las predicciones parecen ser insesgadas en el primer 
periodo (Fig. 4a), debido a que la línea de regresión casi coincide con la línea de predicción perfecta. Sin embargo en el periodo más reciente, la figura sugiere estimaciones con algún grado de sesgo, al aparecer la línea de regresión sistemáticamente por debajo de la línea de 45ํㅡ, lo que sugiere la existencia de predicciones optimistas, con proyecciones de crecimiento sistemáticamente superiores a las efectivamente observadas en la realidad. Puede notarse que en los meses de May-00, September-00, January-01, May01, September-01, January-02 y May-02 se realizó predicciones de muy largo plazo (19m, 23m y 27m), con errores elevados, lo que puede incrementar la dispersión.

Fig. 4a. 1991-2008

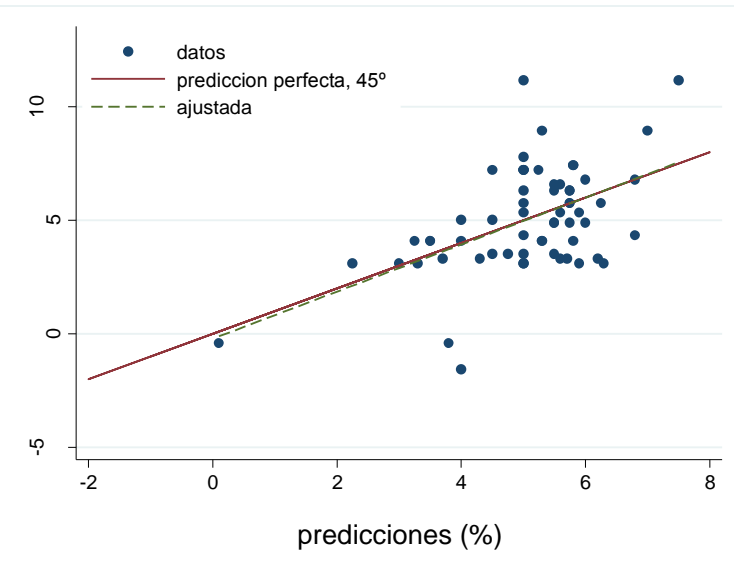

Fig. 4b. 2009-2016

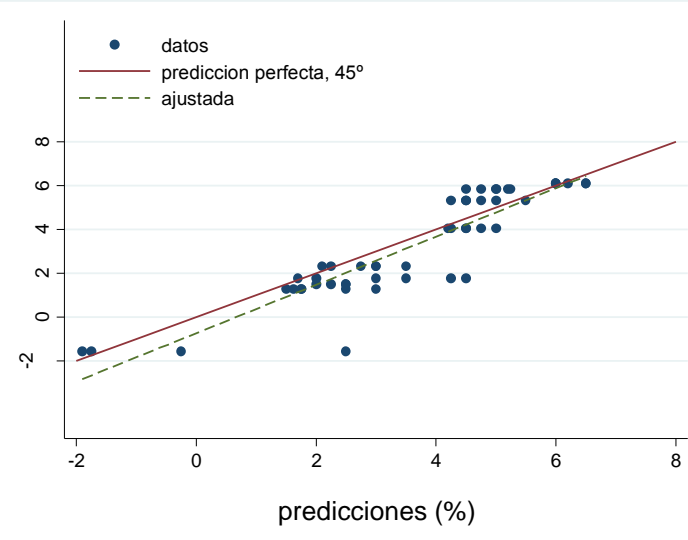

Fig. 4: Predicciones versus realizaciones, según el periodo en el que se realizó la predicción (ver Tabla 4).

\section{Precisión de las Predicciones}

En la Tabla 2 se reportan estimaciones de error en los pronósticos visualizados en las Fig. 4a y 4b. Se tienen las estimaciones globales (111 observaciones) de los errores de las predicciones realizadas en todos los años y para todos los horizontes. En la segunda parte se muestran los errores de predicciones realizadas en el periodo total, pero separando el horizonte en corto plazo ( 3,6 y 7 meses) y en largo plazo (11,12 y 15 meses). Finalmente, la tabla muestra los errores para todos los horizontes de predicción, pero separando las predicciones realizadas en 1991-2008 y en 2009-2016. La tabla sugiere que:

a) Respecto al error promedio de predicción (ME, e = a - f), su signo indica optimismo/pesimismo en las predicciones. Globalmente ME es negativo (-0.185), lo que sugiere en promedio predicciones optimistas $(a<f)$, lo que está de acuerdo con la observación previa a partir de la Fig. 2. Por otro lado, en las predicciones de corto plazo el ME $>0$, sugiriendo expectativas promedio pesimistas, a diferencia de las predicciones de largo plazo, donde parecen predominar el optimismo $(\mathrm{ME}<0)$. Respecto de los subperiodos, en ambos casos el ME<0, aunque su valor en 1991-2008 fue bastante menor que en el periodo 2009-2016, lo que sugiere que en periodo más reciente predominaron las predicciones optimistas (sobrevaloradas). Esto es consistente con el análisis gráfico previo.

b) Respecto a los demás indicadores de errores, la Tabla indica que las estimaciones parecen ser más precisas en el corto plazo (3-6-7 meses) que en el largo plazo (11-12-15), como se esperaba. En efecto, MAE aumenta de 0.870 a 1.528, mientras que RMSE aumenta de 1.147 a 2.088 . Por otro lado, los errores de predicción en promedio han ido disminuyendo a través del tiempo, comparando el sub-periodo 19912008 con el periodo 2009-2016. Aquí el MAE cae de 1.496 a 0.775, el RMSE cae de 1.969 a 1.074.

Tabla 2: Errores por Periodo de Realización de la Predicción, y por Horizonte

\begin{tabular}{|l|c|c|c|c|}
\hline & Num. obs. & ME & MAE & RMSE \\
\hline Periodo total y horizonte total & 111 & $(0.185)$ & 1.171 & 1.628 \\
\hline Horizonte corto plazo 03-06-07 & 43 & 0.250 & 0.870 & 1.147 \\
\hline Horizonte largo plazo 11-12-15 & 43 & $(0.355)$ & 1.528 & 2.088 \\
\hline Periodo 1991-2008 & 61 & $(0.033)$ & 1.496 & 1.969 \\
\hline Periodo 2009-2016 & 50 & $(0.370)$ & 0.775 & 1.074 \\
\hline
\end{tabular}




\section{Insegamiento y Eficiencia Débil de los Pronósticos}

Anteriormente se señaló que el rechazo de la hipótesis nula conjunta $H_{0}: \beta_{0}=0$ y $H_{0}: \beta_{1}=1$ indica la presencia de sesgo débil e ineficiencia débil en los pronósticos. La Tabla 3 muestra las estimaciones de los datos visualizados en las Fig. 4a y 4b, utilizando el procedimiento estándar clásico, consistente en evaluar conjuntamente el intercepto y pendiente de la regresión con errores estándares corregidos por Newey-West. También se muestran las pruebas de significancia individual de intercepto y de pendientes, por separado.

Como resultado, la Tabla 3 muestra que en el corto plazo la regresión sugiere insesgamiento, pero que en las predicciones de largo plazo aparece sesgo. Esto puede ser razonable, debido a que en el corto plazo es justamente los pronósticos se esperan más precisos. También se rechaza la eficiencia en el corto plazo, pero no se rechaza en el largo plazo, aunque el estadístico en ambos casos no es demasiado diferente.

Por otro lado, las estimaciones sugieren que en el sub-periodo 2009-2016 se evidenció mayores problemas en las predicciones, debido a que se rechaza la hipótesis conjunta de eficiencia débil al $5 \%$, y al mismo tiempo se rechaza la hipótesis de insesgamiento. Esto llama la atención por cuanto, como se vio anteriormente, fue en este periodo donde las estimaciones tuvieron menor error de predicción (en base al MAE y RMSE, por ejemplo). Debido justamente a esto es que en la Tabla aparece insesgamiento en el periodo 1991-2008, y sesgo en el sub-periodo 2009-2016. Gráficamente puede confirmarse esto comparando la Fig. 4a versus la Fig. 4b. La Tabla 4 muestra los resultados de las regresiones base que permitieron construir la Tabla 3.

Tabla 3: Estimaciones de Insesgamiento y Eficiencia Débil

Hipótesis nula conjunta $H_{0}: \beta_{0}=0$ y $H_{0}: \beta_{1}=1$; * indica rechazo de $\mathrm{Ho}$ al $95 \%$

\begin{tabular}{|c|c|c|c|}
\hline & $\begin{array}{c}\text { Test Conjunto } \\
\text { (intercep=0 y pend=1) }\end{array}$ & $\begin{array}{c}\text { Test de Insesgamiento } \\
\text { (intercep=0) }\end{array}$ & $\begin{array}{l}\text { Test de Eficiencia } \\
\quad(p e n d=1)\end{array}$ \\
\hline Periodo Total & $\begin{array}{c}F(2,109)=2.74 \\
\text { Prob }>F=0.0689\end{array}$ & $\begin{array}{c}F(1,109)=4.68 \\
\text { Prob }>F=0.0328^{*}\end{array}$ & $\begin{array}{c}F(1,109)=2.18 \\
\text { Prob }>F=0.1423\end{array}$ \\
\hline Corto plazo 03-06-07 & $\begin{array}{c}F(2,41)=2.35 \\
\text { Prob }>F=0.1083\end{array}$ & $\begin{array}{c}F(1,41)=2.61 \\
\text { Prob }>F=0.1139\end{array}$ & $\begin{array}{c}F(1,41)=4.39 \\
\text { Prob }>F=0.0424^{*}\end{array}$ \\
\hline Largo plazo 11-12-15 & $\begin{array}{c}F(2,41)=2.81 \\
\text { Prob }>F=0.0721\end{array}$ & $\begin{array}{c}F(1,41)=5.16 \\
\text { Prob }>F=0.0284^{*}\end{array}$ & $\begin{array}{c}F(1,41)=3.90 \\
\text { Prob }>F=0.0549\end{array}$ \\
\hline $1991-2008$ & $\begin{array}{c}F(2,59)=0.03 \\
\text { Prob }>F=0.9692\end{array}$ & $\begin{array}{c}F(1,59)=0.05 \\
\text { Prob }>F=0.8180\end{array}$ & $\begin{array}{c}F(1,59)=0.04 \\
\text { Prob }>F=0.8455\end{array}$ \\
\hline $2009-2016$ & $\begin{array}{c}F(2,48)=4.13 \\
\text { Prob }>F=0.0222^{*}\end{array}$ & $\begin{array}{c}F(1,48)=6.37 \\
\text { Prob }>F=0.0149^{*}\end{array}$ & $\begin{array}{c}F(1,48)=2.37 \\
\text { Prob }>F=0.1305\end{array}$ \\
\hline
\end{tabular}

Tabla 4: Regresión con errores estandar de Newey-West.

La regresión general es: $A_{p}=\beta_{0}+\beta_{1} F_{t+h, p}+e_{t+h, p} ;{ }^{*} \mathrm{p}<0.05 ;{ }^{* *} \mathrm{p}<0.01$

\begin{tabular}{cccccc}
\hline & $\begin{array}{c}\text { Periodo } \\
\text { Total }\end{array}$ & $\begin{array}{c}\text { corto plazo } \\
(3-6-7 \text { meses })\end{array}$ & $\begin{array}{c}\text { largo plazo } \\
(11-12-15 \text { meses })\end{array}$ & $\begin{array}{c}1991- \\
2008\end{array}$ & $\begin{array}{c}2009- \\
2016\end{array}$ \\
\hline pend & 1.108 & 1.179 & 1.441 & 1.036 & 1.103 \\
& $(15.14)^{* *}$ & $(13.80)^{\star *}$ & $(6.45)^{\star *}$ & $(5.69)^{\star *}$ & $(16.49)^{* *}$ \\
\multirow{2}{*}{ cons } & -0.659 & -0.517 & -2.409 & -0.214 & -0.736 \\
& $(2.16)^{*}$ & $(1.62)$ & $(2.27)^{*}$ & $(0.23)$ & $(2.52)^{*}$ \\
$\mathrm{~N}$ & 111 & 43 & 43 & 61 & 50 \\
\hline
\end{tabular}

\section{Insegamiento y Eficiencia Fuerte de los Pronósticos}

La Tabla 5 muestra las estimaciones de insesgamiento fuerte, tal como se señaló en la metodología, consistente en una prueba estadística de que la media de los errores es cero, basado en una regresión con errores estándares de Newey-West. Aquí puede observarse que la hipótesis de insesgamiento fuerte es rechazado solamente para el periodo 2009-2016 (t-value: -2.57). Recordemos que ahora Ho: insesgamiento fuerte (que promedio de los errores es cero). Esto confirma el resultado obtenido en la Tabla 3 para este sub-periodo. Para el caso global, anteriormente se había detectado sesgo débil, pero sólo al $3.28 \%$ de significancia. Ahora la Tabla 5 indica insesgamiento fuerte para el periodo completo. 
Tabla 5: Insesgamiento fuerte: Test de media cero de los errores

Newey-West standard errors; Ho:_cons=0; insesgamiento fuerte; * $p<0.05 ;{ }^{* *} p<0.01$

\begin{tabular}{cccccc}
\hline & Periodo & corto plazo & largo plazo & $1991-$ & $2009-$ \\
& Total & $3-6-7$ meses & $11-12-15$ meses & 2008 & 2016 \\
\hline cons & -0.185 & 0.250 & -0.355 & -0.033 & -0.370 \\
t test & $(1.20)$ & $(1.45)$ & $(1.12)$ & $(0.13)$ & $(2.57)^{*}$ \\
$\mathrm{~N}$ & 111 & 43 & 43 & 61 & 50 \\
\hline
\end{tabular}

La Tabla 6 muestra los resultados de una prueba de eficiencia fuerte, de acuerdo a lo señalado en el apartado metodológico. Para ello se incluyó los errores de predicción previos como variables explicativas de una regresión con errores estándares de Newey-West. La Tabla sugiere que las predicciones fueron eficientes, en el sentido de que los errores de predicción previos a través de una especificación Moving Average (recogido en el coeficiente de la variable ARMA L.ma) no permiten mejorar las predicciones del periodo siguiente. En específico, la hipótesis nula es existencia de eficiencia fuerte en las predicciones, es decir Ho: "eficiencia fuerte". Esto equivale a que los coeficientes ARMA L.ma no son estadísticamente significativos en la regresión, lo que se verifica cuando el estadístico $t<|2.0|$ para el coeficiente ARMA L.ma. Con esto se tiene alguna evidencia que, al momento de realizar una predicción, la información útil que pudiera estar contenida en los errores de predicción previos, ya estuvo incluida en la predicción del BCCh.

Tabla 6: Regresión con errores estandar de Newey-West, t-values entre paréntesis; * $p<0.05 ;{ }^{* *} p<0.01$

\begin{tabular}{cccccc}
\hline & Periodo & corto plazo & largo plazo & $1991-$ & $2009-$ \\
& Total & $3-6-7$ meses & $11-12-15$ meses & 2008 & 2016 \\
\hline $\mathrm{f}$ & 1.107 & 1.172 & 1.467 & 1.070 & 1.103 \\
& $(8.95)^{\star *}$ & $(12.06)^{\star *}$ & $(4.16)^{\star *}$ & $(3.44)^{\star *}$ & $(12.97)^{\star *}$ \\
\multirow{2}{*}{ _cons } & -0.651 & -0.487 & -2.525 & -0.391 & -0.736 \\
& $(1.03)$ & $(0.94)$ & $(1.49)$ & $(0.23)$ & $(2.13)^{\star}$ \\
\hline \multirow{2}{*}{ ARMA L.ma } & 0.087 & -0.099 & 0.173 & 0.108 & 0.011 \\
& $(0.97)$ & $(0.61)$ & $(0.99)$ & $(0.75)$ & $(0.06)$ \\
sigma_cons & 1.600 & 1.058 & 1.969 & 1.956 & 0.990 \\
& $(20.67)^{\star *}$ & $(7.36)^{\star *}$ & $(10.39)^{\star *}$ & $(12.37)^{\star *}$ & $(11.70)^{\star *}$ \\
$\mathrm{~N}$ & 111 & 43 & 43 & 61 & 50 \\
\hline
\end{tabular}

\section{DISCUSIÓN}

El presente estudio provee de estimaciones de precisión, insesgamiento y eficiencia en las predicciones de crecimiento económico realizadas por el Banco Central de Chile desde la creación del Banco hasta la fecha. En este sentido se trata del estudio más amplio al respecto. Actualmente existen sólo dos estudios que han realizado estimaciones similares para Chile: Albagli et al. (2003) y Pincheira (2010). Existen otros estudios que analizan predicciones del Banco Central, aunque de otras variables como inflación e IMACEC. Albagli et al. (2003) realizó sólo estimaciones de precisión, y encuentran que esta ha ido mejorando a través del tiempo, un resultado que en general coincide con lo que nosotros hemos obtenido, a pesar de que nuestro estudio incluye un periodo mucho más extenso, no considerado por esos autores. Estos autores sólo calcularon medidas de precisión, cuyos resultados fueron similares a los que nosotros obtuvimos. Sin embargo, Albagli et al. (2003) no realizaron pruebas de insesgamiento ni de eficiencia, tal como nosotros lo hicimos aquí.

Pincheira (2010) analizó precisión y eficiencia, sin hacer mediciones de un posible sesgo. Encontró que los pronósticos del Banco Central de Chile pueden mejorar a la luz de las estimaciones, puesto que han sido superados por pronósticos alternativos (de otras instituciones privadas). A pesar de que dicho estudio analizó básicamente nuestro primer subperiodo (1991-2009), esto también coincide en lo general con nuestros resultados. Pincheira (2010) realizó pruebas de eficiencia, cuyos resultados en lo general coinciden con los nuestros. En adición, nosotros incluimos pruebas de insesgamiento, las que no fueron realizadas por Pincheira (2010). Respecto a la evidencia internacional, esta es bastante mixta. Predicciones inadecuadas de crecimiento del PIB se han detectado también en los EEUU por Sinclair et al. (2008), y por Clements et al. (2007), quienes encuentran a través de diferentes enfoques que hay evidencia de sesgo sistemático en las predicciones. En Inglaterra, Bank of England (2015) no encuentra evidencia significativa de sesgo en los pronósticos de corto y mediano plazo, aunque para largo plazo encuentran sesgo positivo e ineficiencia. Recientemente Ruiz Díaz (2017) comparó las estimaciones de 19 economías de América Latina y El Caribe, para el período 2003-2013, concluyendo que, contrario a la idea de que las proyecciones económicas de las organizaciones oficiales tienden a ser optimistas (o sesgadas positivamente), encontraron que las proyecciones del FMI y CEPAL fueron sesgadas hacia abajo. En Latinoamérica, Capistrán y LópezMoctezuma (2010) encuentran que los pronósticos de consenso (externos) parecen ser, en general, más precisos que los pronósticos del Banco de México. 


\section{CONCLUSIONES}

Los principales resultados del estudio sugieren que predominó un sesgo optimista en las predicciones de crecimiento económico del Banco Central de Chile. Este es un resultado bastante plausible, dada la evidencia de otros estudios internacionales que obtienen similares sesgos en el otros Bancos Centrales. Nuestros resultados indican que los errores medios de las predicciones de crecimiento económico realizadas por el Banco Central de Chile fueron mayores en las predicciones de largo plazo, como se esperaba. Por otro lado, en las predicciones de crecimiento de corto plazo, los sesgos promedio fueron más bien pesimistas. Finalmente, si bien las predicciones realizadas en el periodo 1991-2008 fueron menos precisas, en promedio fueron insesgadas y eficientes, a diferencia de las realizadas en el periodo 2009-2016, que mostraron poseer un sesgo optimista estadísticamente significativo. Esto, a pesar de que el ajuste estadístico en las Fig. 4a y 4b parece ser mejor en el subperiodo 2009-2016 que en periodo 1991-2008, a pesar de la inestabilidad económica de ese periodo debido a las consecuencias de la crisis inmobiliaria de los EEUU.

Esto permite concluir que las predicciones globales del Banco Central de Chile tienen espacio para mejorar, tanto en eficiencia como en insesgamiento. Sin embargo, la evidencia internacional comparada muestra que en promedio este tipo de problemas en eficiencia como en insesgamiento son comunes en los bancos centrales de otros países, y que por lo tanto en Banco Central de Chile parece no encontrarse muy lejos del estándar internacional a este respecto. El mejoramiento de la calidad de estas predicciones es de interés, como mencionamos antes, para Congreso respecto por ejemplo de la definición del presupuesto global de la nación, para el Gobierno para la planificación de políticas fiscales, para el propio BCCh para diseñar políticas monetarias, y en general para todos los agentes económicos que toman decisiones de inversión o consumo en un ambiente de incertidumbre.

\section{REFERENCIAS}

Albagli, E., G. Contreras, P. García, I. Magendzo, R. Valdés, Errores de Proyección en Perspectiva, Documentos de Trabajo No 199, Santiago, Banco Central de Chile (2003)

Bank of England, Evaluating Forecast Performance, Independent Evaluation Office, November (2015)

Capistrán, C. y G. López-Moctezuma, Las expectativas macroeconómicas de los especialistas. Una evaluación de pronósticos de corto plazo en México, El Trimestre Económico, LXXVII (2), 275-312 (2010)

Croushore, D., An Evaluation of Inflation Forecasts from Surveys Using Real-Time Data, The B.E. Journal of Macroeconomics, 10(1), 1-32 (2010)

Díaz Ruiz, G., Are official GDP growth forecasts for Latin American and Caribbean countries downward biased? https://doi.org/10.1016/j.cesjef.2016.10.001, Cuadernos de Economía, 40(114), 236-246 (2017)

Granger, C. y P. Newbold, Spurious Regressions in Econometrics, England, University of Nottingham (1963)

Higgins, M., Advances in Economic Forecasting, Kalamazoo, Mich, W.E. Upjohn Institute (2011)

Holden, K., D.A. Peel y J. L. Thompson, Economic Forecasting: An Introduction. Cambridge University Press- Business \& Economics (1990)

Lahiri, K. y X. Sheng, Learning and heterogeneity in GDP and inflation forecasts, International Journal of Forecasting 26 : 265-292 (2010)

Mankiw, N.G. y M. D. Shapiro, Do We Reject Too Often? Small Sample Bias in Tests of Rational Expectations Models, Economics Letters, 20,139-145 (1986)

Mincer, J. y V. Zarnowitz, The Evaluation of Economic Forecast, New York, New York NBER (1969)

Newey, W.K. y K.D. West, A Simple, Positive Semi-definite, Heteroskedasticity and Autocorrelation Consistent Covariance Matrix, Econometrica, 55(3), 703-708 (1987)

Pincheira, P., A real time evaluation of the Central Bank of Chile GDP Growth Forecasts, Santiago, Central Bank of Chile, Documentos de Trabajo 556 (2010)

Sinclair, T., H. Stekler y F. Joutz, Are Unbiased Forecasts Really Unbiased? Another Look at the Fed Forecast, New York: Institute for International Economic Policy (2008)

Theil, H., Applied Economic Forecasting, North-Holland: Amsterdam (1966)

Villada, F., N. Muñoz y E. García-Quintero, Redes Neuronales Artificiales aplicadas a la Predicción del Precio del Oro, http://dx.doi.org/10.4067/S0718-07642016000500016, Información tecnológica, Inf. Tecnol., 27 (5), $143-150$ (2016)

Webb, Roy H., The Irrelevance of Tests for Bias in Series of Macroeconomic Forecasts, Federal Reserve Bank of Richmond Economic Review, November/December, 3-9 (1987) 
